\title{
RESEARCH HIGHLIGHT OPEN SARS-CoV-2 spike protein: a key target for eliciting persistent neutralizing antibodies
}

\author{
Yang Yang ${ }^{1,2,3}$ and Lanying $\mathrm{Du}^{4}$ \\ Signal Transduction and Targeted Therapy (2021)6:95
}

; https://doi.org/10.1038/s41392-021-00523-5
A recent paper published in Science describes the detection of IgG antibody responses in individuals infected by severe acute respiratory syndrome coronavirus (SARS-CoV-2). The authors also examined the duration of antibody production and the correlation between lgG antibody titers and neutralizing antibody titers. ${ }^{1}$ This study provides information about the kinetics of antibody production, and the functionality and longevity of these antibodies, in patients with Coronavirus Disease 2019 (COVID-19).

The SARS-CoV-2 genome encodes spike (S), nucleocapsid, membrane, and envelope structural proteins. The $S$ protein plays a key role in viral infection and pathogenesis. ${ }^{2}$ It comprises subunits S1 and S2: S1 harbors the N-terminal domain (NTD) and the receptor-binding domain (RBD), whereas S2 harbors heptad repeat 1 (HR1) and HR2 (Fig. 1a). SARS-CoV-2 infection undergoes a series of processes: the RBD first binds its receptor, angiotensin converting enzyme 2 (ACE2), to form an RBD/ACE2 complex. This triggers conformational changes in the $S$ protein, leading to membrane fusion mediated via HR1 and HR2; this process culminates in viral entry into target cells (Fig. 1b). Different from other structural proteins, the $\mathrm{S}$ protein is a critical target for the induction of antibodies, particularly neutralizing antibodies, specific for SARS-CoV-2. Antibodies targeting various regions of $S$ protein have different mechanisms in inhibiting SARS-CoV-2 infection. For example, NTD-targeting antibodies (monoclonal antibodies (mAbs) or their fragments) bind the NTD to form an NTD/mAb complex, thereby preventing conformational changes in the $S$ protein and blocking membrane fusion and viral entry (Fig. 1b). By contrast, RBD-targeting antibodies such as mAbs and nanobodies (Nbs) form $\mathrm{RBD} / \mathrm{mAb}$ or $\mathrm{RBD} / \mathrm{Nb}$ complexes that inhibit binding of the RBD to ACE2, thereby preventing entry of SARS-CoV-2 into target cells (Fig. 1b). Thus, understanding the aforementioned mechanism underlying SARS-CoV-2 infection and the mode of action of anti-SARS-CoV-2-S antibodies will help elucidate the kinetics of antibody production in SARS-CoV-2infected individuals, and facilitate the development of effective countermeasures. In general, antibodies targeting the viral RBD are more potent than the antibodies targeting other regions (such as NTD) of S protein, but they might be less broad in inhibiting multiple virus strains.

The Science paper shows that anti-SARS-CoV-2-S antibodies are elicited at detectable titers after infection. ${ }^{1}$ The authors detected IgG antibodies in convalescent plasma from patients with mild-tomoderate COVID-19 symptoms. They performed an ELISA by coating plates with a stabilized SARS-CoV-2 S trimer protein.
Screening 72,401 samples yielded a positive rate of $41.5 \%(30,082 /$ 72,201 ), with IgG titers of $\geq 1: 80$; the majority of positive samples contained moderate $(1: 320$ or $1: 960)$ to high $(1: 2880)$ titers of S-specific antibodies.

They then selected 120 plasma samples with lgG titers varying from non-detectable to $\geq 1: 2880$ and examined neutralizing activity against authentic SARS-CoV-2 infection. Plasma with IgG titers of 1:320, 1:960, or $\geq 1: 2880$ corresponded to neutralizing antibody titers of 1:30, 1:75, and 1:550, respectively, and all samples with lgG titers ranging from 1:960 to $\geq 1: 2880$ neutralized SARS-CoV-2 infection. Overall, these results indicate that SARSCoV-2 S-targeting IgG antibodies in SARS-CoV-2-infected patients neutralized authentic SARS-CoV-2 infection, and that the induced IgG antibody titer correlated with the neutralizing antibody titer. The data from this study are consistent with those from previous studies on SARS-CoV and Middle East respiratory syndrome coronavirus (MERS-CoV), other coronaviruses with high fatality rates in humans; the latter studies show that, virus-specific antibodies, particularly those targeting $S$ proteins and/or RBD fragments, correlate positively with neutralizing antibody titers. ${ }^{2}$ In addition, the Science paper also examined longevity of IgG antibodies specific for the SARS-CoV-2 S protein in 121 volunteers over a period of around 5 months, separated by three mean time points (30, 82, and 148 days after symptom onset). ${ }^{1}$ Notably, specific antibodies were maintained for at least 5 months, showing a modest decrease over time (particularly at initial titers of $1: 960$ to $\geq 1: 2880$ ). Moreover, neutralizing antibody titers still correlated with neutralizing antibody titers at Day 148 . As pointed out by the authors, detection and measurement of antibodies generated by SARS-CoV-2 infection should be extended over a longer period of time. This may give indications as to whether people infected with SARS-CoV-2 can be reinfected and, if so, at which antibody level. In the case of SARS-CoV and MERS-CoV, high-titer S/RBD-specific IgG and neutralizing antibodies can be maintained for up to 12 months post-vaccination, providing complete protection against viral infection. ${ }^{2}$ It is therefore expected that the higher the titer of specific IgG antibodies with neutralizing activity against SARS-CoV-2 infection, and the longer they persist, the more effective they will be in preventing reinfection.

IgG antibodies induced by SARS-CoV-2 in patients with moderate-to-severe symptoms mainly target the $\mathrm{S}$ protein; ${ }^{1}$ however, it is unclear which regions (RBD, NTD, or other fragments) of the $S$ protein are targeted, or whether the induced

\footnotetext{
${ }^{1}$ Roy J. Carver Department of Biochemistry, Biophysics and Molecular Biology, lowa State University, Ames, IA, USA; ${ }^{2}$ Howard Hughes Medical Institute, Yale University, New

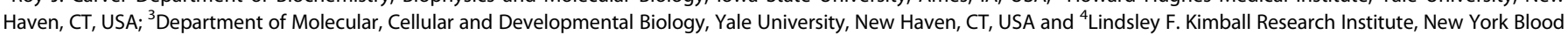
Center, New York, NY, USA

Correspondence: Lanying Du (Idu@nybc.org)
}

Received: 7 December 2020 Revised: 26 January 2021 Accepted: 28 January 2021

Published online: 26 February 2021 


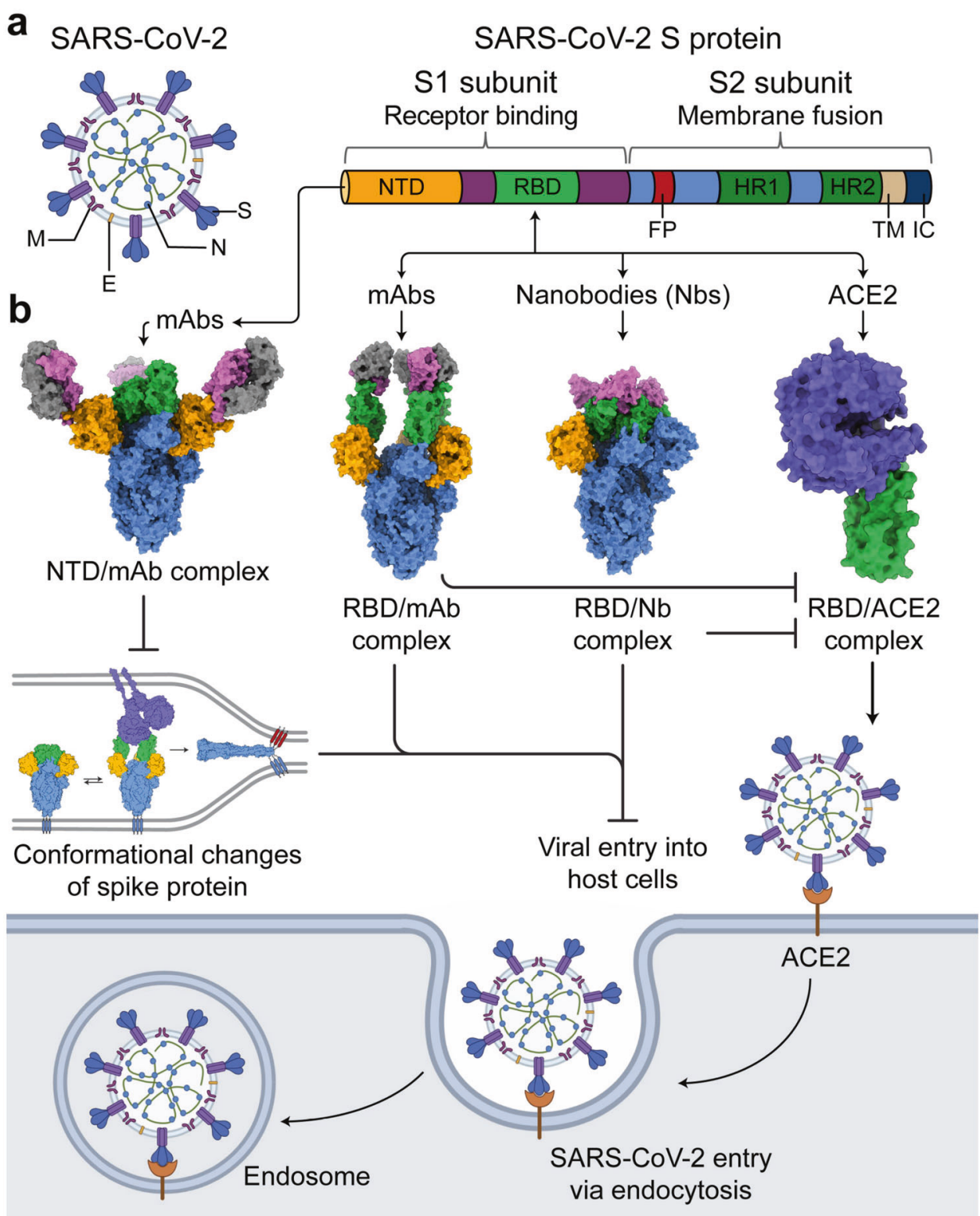

Fig. 1 SARS-CoV-2 spike (S) protein is a key target for eliciting neutralizing antibodies. a Schematic structures of SARS-CoV-2 virion and its $S$ protein. M, membrane; $E$, envelope; N, nucleocapsid. Viral RNA is located inside the virion. NTD, N-terminal domain; RBD, receptor-binding domain; FP, fusion peptide; HR1 and HR2, heptad region 1 and 2; TM, transmembrane domain; IC, intracellular tail. b Mode of action of SARSCoV-2 S-specific neutralizing antibodies. Monoclonal antibodies (mAbs) targeting S protein NTD prevent conformational changes of the $S$ protein that are required for S2-mediated membrane fusion, and hence inhibit viral entry into host cells. RBD-targeting neutralizing mAbs or nanobodies (Nbs), on the other hand, bind directly to SARS-CoV-2 S protein RBD and compete with the cellular receptor, angiotensin converting enzyme 2 (ACE2), resulting in neutralization of viral infection and clearance of the virus. The following PDB entries are used for structural illustrations: 7C2L (structure of SARS-CoV-2 S in complex with NTD-targeting mAb 4A8), 7K4N (structure of SARS-CoV-2 S in complex with RBD-targeting mAb S2E12), 7KKK (structure of SARS-CoV-2 S in complex with RBD-targeting nanobody Nb6), and 6VW1 (structure of SARS-CoV-2 RBD in complex with human ACE2). SARS-CoV-2 S NTD is colored in orange, RBD in green, and the rest part of S protein in light blue. ACE2 is colored in purple. This figure was prepared using BioRender (https://biorender.com/) and UCSF ChimeraX (https://www.cgl.ucsf. edu/chimerax/)

antibodies show cross-reactivity and/or cross-neutralizing activity against other coronaviruses. Some studies indicate that SARS-CoV2 RBD-specific lgG antibodies are present in convalescent plasma from COVID-19 patients, and that IgG antibodies specific for RBD and $\mathrm{S}$ (ectodomain) correlate positively with anti-SARS-CoV-2 neutralizing antibody titers. ${ }^{3}$ Other studies demonstrate the generation of persistent, but slightly reduced, lgG antibodies in patients with COVID-19; these antibodies also target SARS-CoV-2 $\mathrm{RBD}$, and the titers correlate with S-specific neutralizing antibody titers. ${ }^{4}$ Moreover, these antibodies may cross-react with the S/RBD of SARS-CoV, thereby cross-neutralizing SARS-CoV infection; however, they show low to no cross-reactivity with lowpathogenic human coronaviruses such as HKU1, 229E, OC43, or NL63. ${ }^{4,5}$ Considering the high sequence similarity between SARSCoV-2, SARS-CoV, and other SARS-like coronaviruses, and their use of the same ACE2 receptor, further studies are needed to understand whether antibodies generated by SARS-CoV-2infected patients cross-react with $S$ proteins or their fragments (S1-RBD/S2), and/or cross-neutralize infections by SARS-like coronaviruses with pandemic potential. 
Studies of highly pathogenic human coronaviruses (SARS-CoV2, SARS-CoV, and MERS-CoV) indicate that, although full-length and fragments (NTD and RBD) of $S$ proteins induce specific antibodies with neutralizing activity, the RBD is a major target for eliciting highly potent neutralizing antibodies with protective efficacy. ${ }^{2}$ The majority of the currently developed anti-SARS-CoV-2 therapeutic antibodies target the $S$ protein, particularly the RBD. Therefore, the S/RBD-specific antibodies have great potential for development as effective therapeutics that prevent COVID-19 spread.

\section{ACKNOWLEDGEMENTS}

This study was supported by the National Institutes of Health $(\mathrm{NIH})$ grants (R01Al157975, R01Al137472, and R01Al139092).

\section{ADDITIONAL INFORMATION}

Competing interests: The authors declare no competing interests.

\section{REFERENCES}

1. Wajnberg, A. et al. Robust neutralizing antibodies to SARS-CoV-2 infection persist for months. Science 370, 1227-1230 (2020).
2. Wang, N., Shang, J., Jiang, S. \& Du, L. Subunit vaccines against emerging pathogenic human coronaviruses. Front. Microbiol. 11, 298 (2020).

3. Salazar, E. et al. Convalescent plasma anti-SARS-CoV-2 spike protein ectodomain and receptor-binding domain IgG correlate with virus neutralization. J. Clin. Invest. 130, 6728-6738 (2020).

4. Iyer, A. S. et al. Persistence and decay of human antibody responses to the receptor binding domain of SARS-CoV-2 spike protein in COVID-19 patients. Sci. Immunol. 5, eabe0367 (2020).

5. Jiang, S. \& Du, L. Effect of low-pathogenic human coronavirus-specific antibodies on SARS-CoV-2. Trends Immunol. 41, 853-854 (2020).

(C) Open Access This article is licensed under a Creative Commons Attribution 4.0 International License, which permits use, sharing, adaptation, distribution and reproduction in any medium or format, as long as you give appropriate credit to the original author(s) and the source, provide a link to the Creative Commons license, and indicate if changes were made. The images or other third party material in this article are included in the article's Creative Commons license, unless indicated otherwise in a credit line to the material. If material is not included in the article's Creative Commons license and your intended use is not permitted by statutory regulation or exceeds the permitted use, you will need to obtain permission directly from the copyright holder. To view a copy of this license, visit http://creativecommons. org/licenses/by/4.0/.

(c) The Author(s) 2021 